\title{
Photodynamics and quantum efficiency of germanium vacancy color centers in diamond
}

\author{
Minh Nguyen, ${ }^{a, t}$ Niko Nikolay, ${ }^{\mathrm{b}, \dagger}$ Carlo Bradac, ${ }^{a}$ Mehran Kianinia, ${ }^{a}$ Evgeny A. Ekimov, ${ }^{\mathrm{c}, \mathrm{d}}$ \\ Noah Mendelson, ${ }^{a}$ Oliver Benson, ${ }^{b}$ and Igor Aharonovich ${ }^{\mathrm{a}, *}$ \\ anniversity of Technology Sydney, School of Mathematical and Physical Sciences, Ultimo, New South Wales, Australia

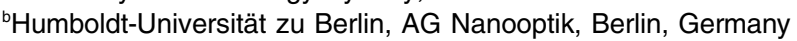 \\ ${ }^{c}$ Russian Academy of Sciences, Institute for High Pressure Physics, Moscow, Troitsk, Russia \\ ${ }^{d}$ Russian Academy of Sciences, Lebedev Physics Institute, Moscow, Russia
}

\begin{abstract}
Color centers in diamond-especially group IV defects-have been advanced as a viable solidstate platform for quantum photonics and information technologies. We investigate the photodynamics and characteristics of germanium-vacancy $(\mathrm{GeV})$ centers hosted in high-pressure high-temperature diamond nanocrystals. Through back-focal plane imaging, we analyze the far-field radiation pattern of the investigated emitters and derive a crossed-dipole emission, which is strongly aligned along one axis. We use this information in combination with lifetime measurements to extract the decay rate statistics of the GeV emitters and determine their quantum efficiency, which we estimated to be $\sim(22 \pm 2) \%$. Our results offer further insight into the photodynamic properties of the GeV center in nanodiamonds and confirm its suitability as a desirable system for quantum technologies.
\end{abstract}

Keywords: nanodiamond; germanium vacancy; color center; quantum efficiency.

Received Oct. 31, 2019; accepted for publication Dec. 11, 2019; published online Dec. 26, 2019.

(C) The Authors. Published by SPIE and CLP under a Creative Commons Attribution 4.0 Unported License. Distribution or reproduction of this work in whole or in part requires full attribution of the original publication, including its DOI.

[DOI: 10.1117/1.AP.1.6.066002]

\section{Introduction}

Single emitters in diamond, especially group IV color centers, have been identified as suitable hardware for many solid-statebased quantum applications-including quantum networks, quantum information processing, and sensing. ${ }^{1-10}$ This is largely due to their favorable optical properties, such as narrow zerophonon line (ZPL) emission and low spectral diffusion. ${ }^{11-15}$ These characteristics arise from the split-vacancy $D_{3 \mathrm{~d}}$ configuration of group IV diamond centers with the foreign atom $(\mathrm{Si}, \mathrm{Ge}, \mathrm{Sn}$, and $\mathrm{Pb})$ occupying an interstitial site between two carbon vacancies along the $\langle 111\rangle$ direction in the diamond lattice. ${ }^{16-22}$ The corresponding inversion symmetry of the defects grants them a vanishing permanent electric dipole moment and

*Address all correspondence to Igor Aharonovich, E-mail: igor.aharonovich@uts .edu.au

†These authors contributed equally to this work. thus renders the optical transition frequency insensitive to firstorder electric-field and strain variations.

Recent studies have focused predominantly on the germaniumvacancy $(\mathrm{GeV})$ defect, which has been successfully engineered using both chemical vapor deposition, high-pressure hightemperature (HPHT), and ion implantation techniques. ${ }^{17,23-26}$ The defect has a zero phonon line around $602 \mathrm{~nm}$, which splits into four lines at cryogenic temperatures. ${ }^{6}$ The successful incorporation of $\mathrm{GeV}$ defects into photonic resonators and plasmonic waveguides has also been demonstrated..$^{27,28}$ However, despite the rapid progress, the photophysical properties of the $\mathrm{GeV}$ centers in nanodiamonds (NDs) are still poorly understood.

In this work, we characterize the photodynamics of $\mathrm{GeV}$ centers hosted in HPHT NDs, both in ensemble and single-emitter measurements. We perform photoluminescence (PL) and lifetime spectroscopy analyses and use Fourier-plane imaging to determine the far-field radiation pattern of the emitters. We then combine these results to produce an approximated estimate of the quantum efficiency $(\mathrm{QE})$ of an ensemble of $\mathrm{GeV}$ emitters. 
We achieve this by mapping the emitters' radiative component $\left(k_{r}\right)$ of their total decay rate $\left(k_{r}+k_{\mathrm{nr}}\right)$ onto changes in the emitters' PL lifetime as we control their dielectric surrounding environment. ${ }^{29,30}$

\section{Materials and Methods}

The NDs containing GeV centers were fabricated via HPHT at the Institute for High Pressure Physics, Russian Academy of Sciences, Moscow. ${ }^{31}$ The process involved the use of naphthalene $\left(\mathrm{C}_{10} \mathrm{H}_{8}\right)$ mixed with germanium, under 8 to $9 \mathrm{GPa}$ of pressure and at temperatures $>1600 \mathrm{~K}$, and resulted in high-quality ND crystals (size $\sim 60 \mathrm{~nm}$ ), containing Ge-related color centers, including germanium-vacancy $(\mathrm{GeV})$ centers.

For characterization, we dispersed the NDs on a transparent glass coverslip $(22 \mathrm{~mm} \times 22 \mathrm{~mm}$, thickness 0.13 to $0.16 \mathrm{~mm}$; Ted Pella), which had been laser scribed with a $5 \times 5$ unit grid consisting of $50 \mu \mathrm{m} \times 50 \mu \mathrm{m}$ squares to facilitate the identification and localization of target emitters. We performed the optical characterization using a lab-built confocal microscope, with confocality achieved via a $4 f$ lens system and a pinhole (diameter $50 \mu \mathrm{m}$ ) positioned within the collection path. Excitation of the emitters and collection of their PL signal was carried out using a 1.4 numerical aperture oil immersion objective. Spectroscopy, lifetime, and second-order autocorrelation measurements were carried out using a spectrometer (Acton SpectraPro, equipped with a PIXIS 256 camera; Princeton Instruments) and two avalanche photodiodes (Count T; Laser Components) arranged in a Hanbury-Brown and Twiss interferometer connected to a correlator (TimeTagger 20; Swabian). We used a 532-nm continuous-wave (cw) laser (SPL-532, Shanghai Dream Lasers Technology) as the primary excitation source for all optical experiments, and we employed a 512-nm pulsed laser (PiL051-FS, Advanced Laser Diode Systems GmbH) for the lifetime measurements. We probed the samples in $\mathrm{cw}$ at an intensity measured at the back aperture of the objective of $500 \mu \mathrm{W}$ while pulsed excitation was performed under an average power of $20 \mu \mathrm{W}$, with a repetition rate of $20 \mathrm{MHz}$. Fourier plane imaging was performed through an additional optical pathway utilizing a $4 f$ lens configuration and $0.5 \times$ magnification at the EMCCD camera (Oxford Instruments iXon Ultra 888). We determined the size of the individual diamond nanoparticles via atomic force microscopy (AFM, XE7; Park Systems).

To fit the back focal plane (BFP) images, we first analytically calculated the sum of two BFP intensity distributions originating from crossed linear dipoles positioned above a glass cover slide using the equations from Ref. 32. The residues to the experimental data were minimized using the sequential least squares programming algorithm with four free parameters: the in-plane angle $(\phi)$ and the out-of-plane angle $(\theta)$ of one dipole, the rotation angle of the perpendicularly oriented dipole, and the relative amplitude of the two dipoles.

\section{Results and Discussions}

We characterized NDs containing either ensembles or single $\mathrm{GeV}$ centers. Figure 1(a) is the AFM image of the investigated NDs, which are $(65.0 \pm 4.4) \mathrm{nm}$, and Fig. 1(b) shows their PL spectrum. The sharp peak at $\sim 602 \mathrm{~nm}$ corresponds to the ZPL emission wavelength of the $\mathrm{GeV}$ center. The inset in Fig. 1(b) displays the second-order autocorrelation measurement, $g^{(2)}(\tau)$, which was used to identify single emitters, $g^{(2)}(0)<0.5$. For this particular emitter, the $g^{(2)}(0)$ value was measured to be $(0.37 \pm 0.02)$, evaluated with a timing jitter of $500 \mathrm{ps}$.

We then performed a detailed analysis of the radiation pattern of $\mathrm{GeV}$ emitters as shown in Fig. 2. Specifically, we used a $4 f$ optical relay system to image the BFP of the objective onto an EMCCD camera (cf. Sec. 2). The corresponding light intensity distribution is representative of the far-field radiation pattern of the emitter and can thus be used to map the in-plane angle $(\phi)$ and the out-of-plane angle $(\theta)$ of the emitting dipole(s) in the sample plane. For each $\mathrm{GeV}$ color center, we expect a crossed-dipole far-field radiation pattern where the dipole moment aligned along the symmetry axis of the defect is dominating. ${ }^{33}$ This, however, has never been shown experimentally thus far. Figure 2 shows the experimental [Fig. 2(a)] and calculated [Fig. 2(b)] BFP radiation pattern for three typical NDs containing each a single $\mathrm{GeV}$ center. The theoretical fit
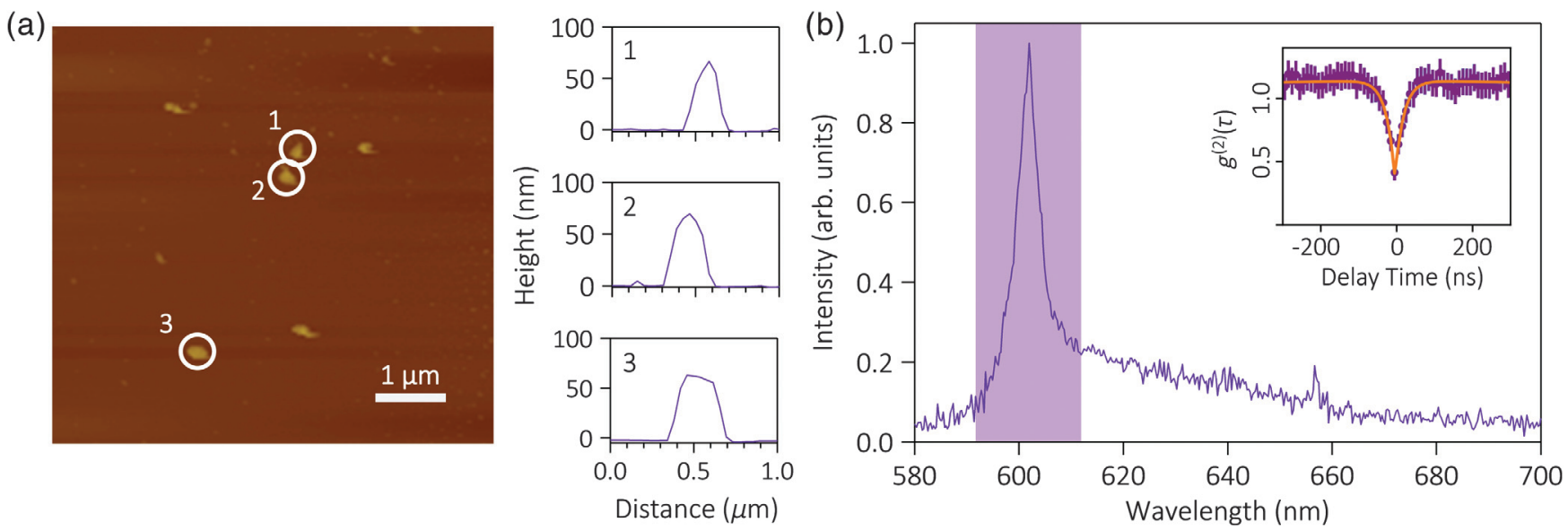

Fig. 1 NDs containing GeV color centers. (a) AFM image showing the typical size of the NDs. The numbered white circles refer to the corresponding height profiles. (b) Characteristic PL spectrum of a typical ND containing GeV color center(s) with ZPL at $602 \mathrm{~nm}$. Inset: second-order autocorrelation function indicating emission from a single $\mathrm{GeV}, g^{(2)}(0)=0.37 \pm 0.02$. The purple shading in the spectrum indicates the band of wavelengths selected by the bandpass filter in the autocorrelation measurement. 
(a)

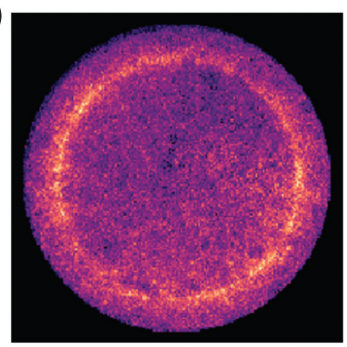

(b)

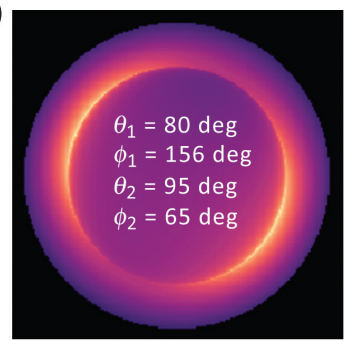

Experimental
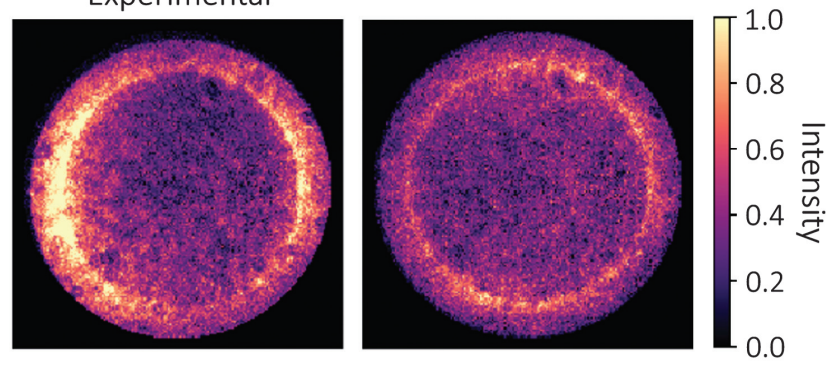

Simulated 2D Dipole
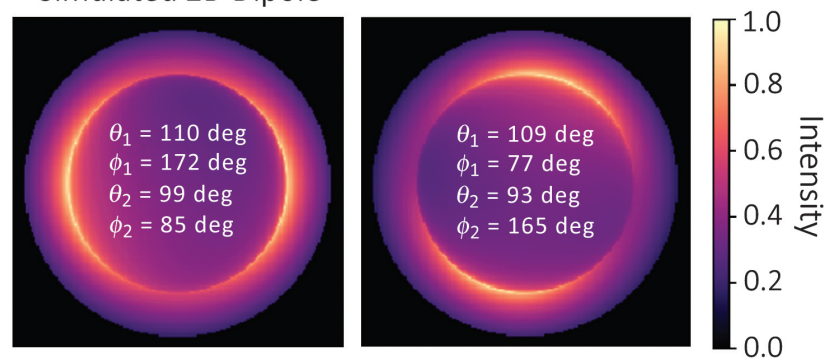

Fig. 2 BFP analysis of three individual GeV single-photon emitters. (a) BFP images measured experimentally for each individual emitter. (b) Corresponding simulated 2-D dipole fits. The fitted in-plane $(\phi)$ and out-of-plane $(\theta)$ angles of the crossed dipole (subscripts 1 and 2) for each GeV center are indicated explicitly.

to the experimental BFP images highlights that the intensity distributions are consistent with the expected crossed-dipole emission, dominated by a single dipole moment. The calculated, best-fit-to-the-data values for the angles $\theta$ and $\phi$ of the crossed dipoles (cf. Sec. 2) are indicated. Since the characterized GeV defects embedded in NDs are randomly distributed with respect to the glass surface, it is expected that both $\theta$ and $\phi$ will vary from crystal to crystal. Note that before each measurement, the single photon nature of the $\mathrm{GeV}$ center was confirmed using second-order autocorrelation measurements.

We also performed an analysis on the emission properties of fluorescent NDs hosting single GeV emitters. Figure 3 illustrates our findings. The photoemission from individual centers is stable [Fig. 3(a)] and reaches saturation intensities of $\sim 10^{5}$ to $10^{6}$ counts/s [Fig. 3(b)]. The single emitter in Fig. 3(b) is representative of the surveyed emitters. It displays a saturation intensity $I_{\infty} \sim 7 \times 10^{5}$ counts $/ \mathrm{s}$ - determined as $I_{\infty}=\left(I_{\infty} P\right) /$ $\left(P_{\text {Sat }}+P\right)$ with $P_{\text {Sat }}$ being the excitation power at saturation. Figure 3(c) summarizes the distribution of lifetimes measured for 18 individual NDs containing GeV centers; Figs. 3(d) and 3(e) show the distribution of their ZPL emission wavelength and full width at half maximum (FWHM). The measured lifetimes varied substantially among centers spanning over the range $\sim 6$ to $25 \mathrm{~ns}$ [Fig. 3(c)], with an average value of (13.8 \pm 2.3) ns and consistent with previous reports. ${ }^{28}$ The lifetime of the $\mathrm{GeV}$ defect in bulk diamond has been measured to be $\sim 1.4$ to 5.5 ns. ${ }^{17,26}$ Considerably long lifetimes in NDs are expected for some color centers, e.g., the nitrogen vacancy (NV) center. $^{34}$ The NV possesses a noninversion symmetry and thus fluctuating electromagnetic fields (e.g., due to traps on the diamond surface) may influence its lifetime significantly. On the other hand, the $\mathrm{SiV}$ defect in NDs exhibits very similar values of measured lifetime ( 1 to $2 \mathrm{~ns}$ ), even in NDs just a few $\mathrm{nm}$ in size. ${ }^{35}$ The fact that the $\mathrm{GeV}$ center shows more variability in lifetime values than the $\mathrm{SiV}$-despite sharing the same crystallographic symmetry - has been suggested to derive from its higher QE. A higher QE would make the $\mathrm{GeV}$ more sensitive to changes in the local photonic density of states, resulting in the lifetime varying more widely from ND to ND.

Finally, we discuss a method to determine the $\mathrm{QE}$ of $\mathrm{GeV}$ centers hosted in diamond nanoparticles. On average, the $\mathrm{GeV}$ center is brighter than the $\mathrm{SiV}$, as was reported by numerous groups. ${ }^{6,26,36}$ However, the value of the QE was never determined experimentally. The method to determine the QE relies on modifying, in a controlled manner, the electromagnetic interaction between the emitters and the surrounding dielectric environment. In general, the total decay rate $(k)$ of an emitter is given by the sum of its radiative $\left(k_{\mathrm{r}}\right)$ and nonradiative $\left(k_{\mathrm{nr}}\right)$ components, with the ratio $k_{\mathrm{r}} / k$ determining the emitter's QE. Of these decay rates, only the radiative one $\left(k_{\mathrm{r}}\right)$ depends on the local density of states (LDOS). We can therefore design an experiment in which we alter deterministically the LDOS and indirectly extract the value of $k_{\mathrm{r}}$ from the corresponding change in the excited state lifetime, $\tau=1 / k .^{30}$ Specifically, for an emitter in an unbounded dielectric medium of refractive index $n$, the transition probability $k_{\mathrm{r}}$ is governed by Fermi's golden rule $k_{\mathrm{r}} \propto n k_{\mathrm{r}}^{0}$, where $k_{\mathrm{r}}^{0}$ is the decay rate in vacuum-so long as the emitter is at a distance $d>\lambda$ from any dielectric interface. For $d<\lambda$, the spontaneous emission rate $k_{\mathrm{r}}$ can be determined from (and strongly depends on) the distance $d$, the dipole orientation (polar angle $\theta$ with respect to the normal to the interface), and the refractive indexes at the interface. Conversely, the nonradiative decay rate $k_{\mathrm{nr}}$ is assumed constant, regardless of $d$. This means that the PL lifetimes measured for the same emitter located close to $(d<\lambda)$ or far from $(d \gg \lambda)$ a dielectric interface should vary due solely to a difference in the rate $k_{\mathrm{r}}$. This ultimately allows for the determination of $k_{\mathrm{r}}, k_{\mathrm{nr}}$, and the emitter QE from simple lifetime measurements. 
(a)

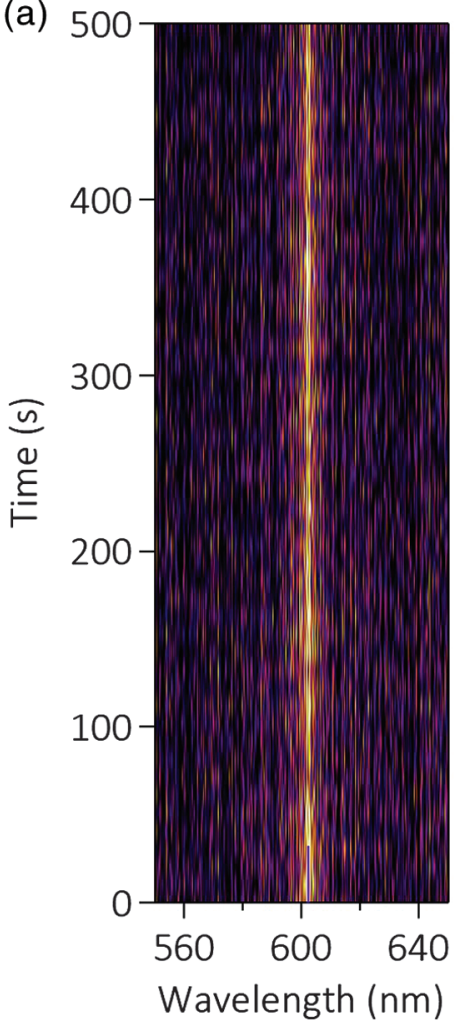

(b)

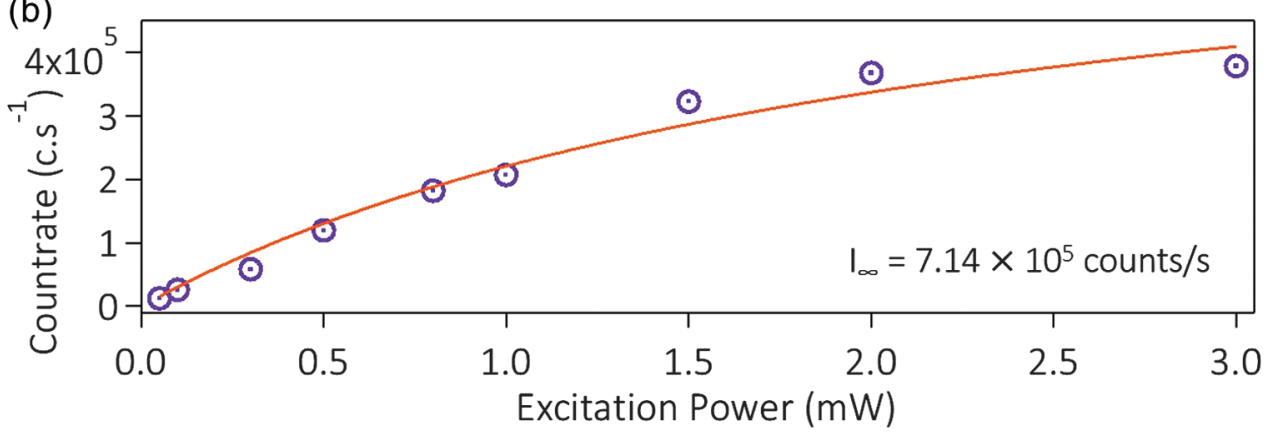

(c)

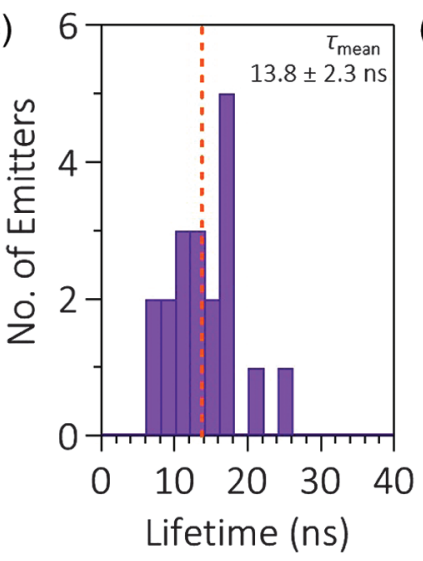

(d)

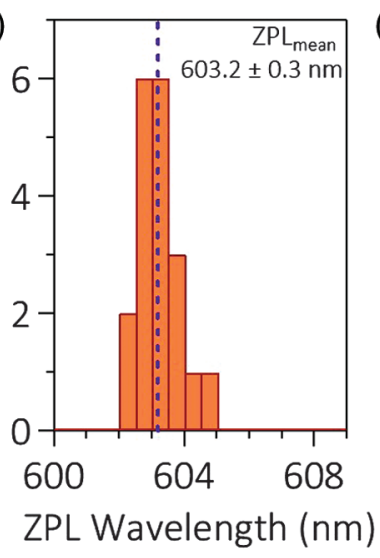

(e)

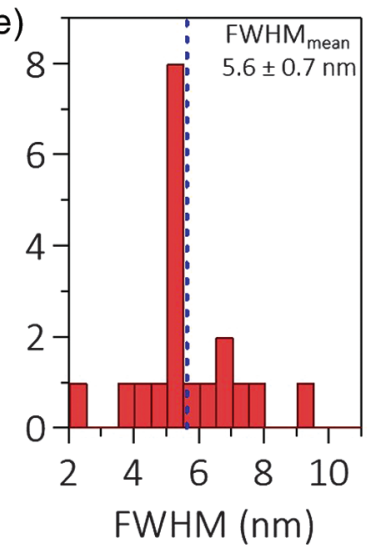

Fig. 3 Summary of the optical properties of characteristic NDs containing GeV centers. (a) Kinetic spectra measurement of a single $\mathrm{GeV}$ emitter, showing photostable emission at $604 \mathrm{~nm}$ (FWHM $\sim 4.27 \mathrm{~nm}$ ) for $500 \mathrm{~s}$ at 10-s integration time intervals. (b) Saturation measurement of the emitter. The extracted saturation intensity is $I_{\infty}=7.14 \times 10^{5}$ counts $/ \mathrm{s}$. (c) Histogram of the emitter lifetime. (d) Histogram of emitter ZPL position. (e) Histogram of FWHMs of the fitted ZPLs. The dotted lines represent the average values of their respective dataset. Each histogram represents a sample size of 18 individual emitters.

Figure 4(a) shows the schematic arrangement of the performed QE measurement. For the first step of the experiment, we prepared a suspension of NDs (average size $s=65 \mathrm{~nm}$ ) in an ethanol-water solution. We dispersed the suspension on a marked glass coverslip (cf. Sec. 2) and measured the lifetime of the GeV(s) in individual NDs. Figure 4(b) shows the characterization of one such ND containing an ensemble of $\mathrm{GeV}$ : the initial measured excited state lifetime is $\tau_{1}=k_{1}^{-1}=(8.3 \pm$ 0.4) ns [Fig. 4(b), purple trace].

For the second step of the experiment, a thick $(\sim 1.5 \mu \mathrm{m})$ layer of polymethyl methacrylate (PMMA) was used as the dielectric material to surround the NDs. Since the glass and the PMMA have a similar refractive index $\left(n_{\mathrm{PMMA}}=1.49\right.$ and $n_{\text {glass }}=1.52$ ), after the PMMA deposition, the NDs can be considered embedded in a homogeneous medium with a refractive index $n \sim 1.5$. The PMMA on top serves the purpose of "removing" the glass-air interface, such that the NDs are effectively considered embedded in an unbounded medium with refractive index $n=1.5$. We repeated the lifetime measurement on the same NDs surveyed in the first step, which we could identify by means of the reference laser-scribed grid on the glass substrate (cf. Sec. 2). Following the PMMA deposition, the measured excited state lifetime is $\tau_{2}=k_{2}^{-1}=(7.1 \pm 0.4) \mathrm{ns}$ [Fig. 4(b), orange trace] - which is shorter than the $\tau_{1}=(8.3 \pm$ 0.4) ns measured at the previous step due to the change in the average radiative decay rate of the emitters. We define the quantity $\beta=k_{2}^{-1} / k_{1}^{-1}=\tau_{2} / \tau_{1}=(0.85 \pm 0.06) \mathrm{ns}$ as the ratio between the overall decay rates after and before the PMMA deposition. We note that the measurement of the lifetime was carried out on an ensemble rather than on a single $\mathrm{GeV}$ center. This was necessary, as the signal-to-noise ratio in the PL lifetime measurement was too low in the second step of the experiment to obtain a meaningful estimate of the lifetime itself, most likely due to the non-negligible PMMA contribution. Specifically, we observed a broad and homogeneous luminescence from the PMMA, which extends over the spectral range 550 to $800 \mathrm{~nm}$, with a count rate approximately half that of the PL signal from the ensemble of emitters. The fact that we used an ensemble rather than an individual $\mathrm{GeV}$ center was taken into account in our analysis (see below). For step two of the measurement, we hypothesize that depositing the PMMA on top of the NDs does not change the nonradiative decay rate $k_{\mathrm{nr}}$ of the hosted $\mathrm{GeV}$ centers.

We cannot directly test this hypothesis experimentally. Nevertheless, considering that (i) diamond color centers are not stable within $\sim 2 \mathrm{~nm}$ from the surface, ${ }^{37}$ (ii) only color centers $\sim 2.5 \mathrm{~nm}$ from the surface might be subject to modifications occurring at the interface, ${ }^{38}$ and (iii) assuming a uniform distribution of $\mathrm{GeV}$ centers in a nanodiamond that, in our case, has an average radius of $32.5 \mathrm{~nm}$, we determine (from geometrical considerations) that only $\sim 4 \%$ of the color centers in a nanoparticle might be affected by any physicochemical change occurring 
(a)

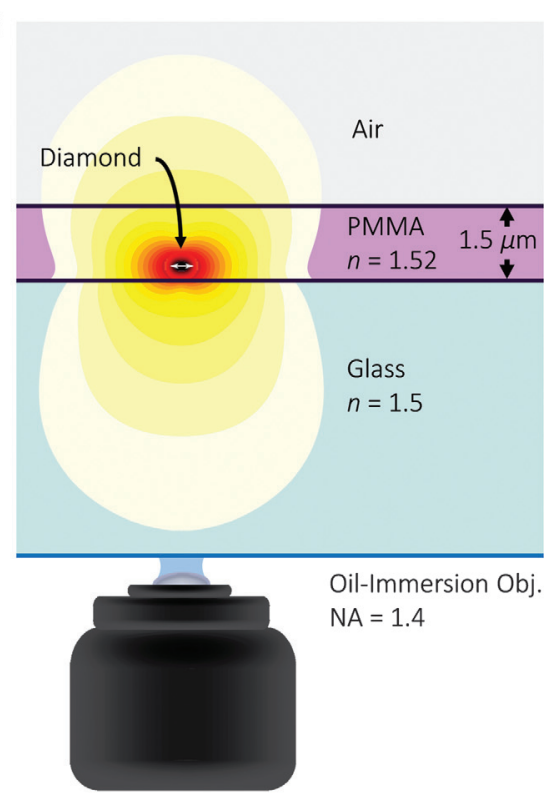

(b)

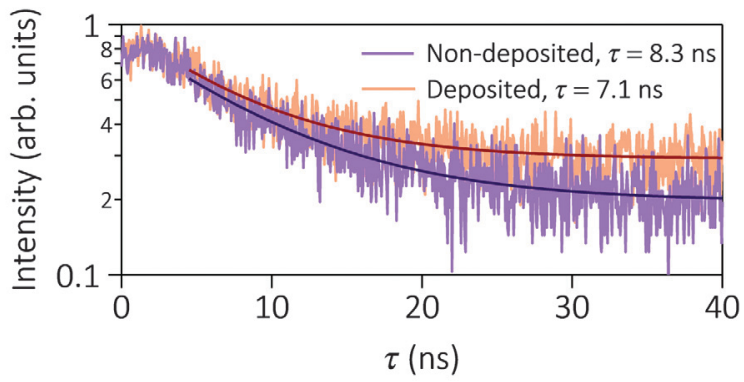

(c)

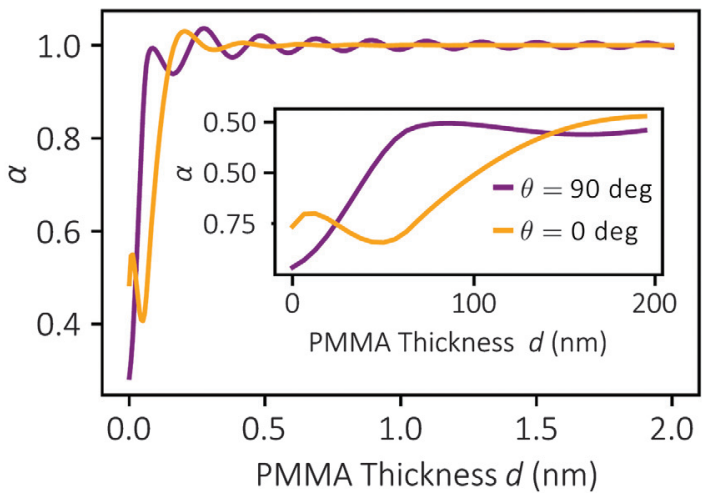

Fig. 4 Measured QE of GeV ensembles. (a) Schematic illustration of the sample: in the first step of the experiment (cf. main text) the NDs lie on a transparent coverslip in air; in the second step of the experiment they are covered with a 1.5- $\mu \mathrm{m}$ thick layer of PMMA acting as an unbound dielectric medium. (b) Filtered emission lifetime measurements before (purple trace) and after (orange trace) deposition of $\sim 1.5 \mu \mathrm{m}$ of PMMA. (c) Simulated values for the ratio between radiative rates as a function of PMMA thickness for parallel (orange trace) and perpendicular (purple trace) dipole orientations. Inset figure shows the simulated values for the range 0 to $200 \mathrm{~nm}$.

locally at the ND-PMMA interface. Additionally, of these centers, (iv) only $\sim 25 \%$ would be subject to interaction with the surface. It follows that about $\sim 1 \%$ of the color centers in the ensemble in each ND would experience an actual change in their nonradiative decay rate $k_{\mathrm{nr}}$ (and hence measured lifetime). Even ignoring (iv), the $4 \%$ error after (i)-(iii) is within the resolution of our lifetime measurement $(\sim 5 \%)$.

Next, using JCMwave (a full vectorial Maxwell solver), we calculate the ratio $\alpha=k_{\mathrm{r} 1} / k_{\mathrm{r} 2}$. This solver allows for the calculation of the power $P$ radiated by a dipole, which is proportional to the radiative decay rate, $k_{\mathrm{r}} \propto P$, and thus gives $\alpha=P_{1} / P_{2}$. Figure 4(c) shows the calculated $\alpha$ as the thickness of the PMMA layer varies in the range 0 to $1.5 \mu \mathrm{m}$. Note that $\alpha$ is determined both for the in-plane $\left(\theta=90 \mathrm{deg}\right.$, denoted as $\left.\alpha_{\|}\right)$ and the out-of-plane ( $\phi=0 \mathrm{deg}$, denoted as $\left.\alpha_{\perp}\right)$ dipole orientations. This is necessary as our lifetime measurements are carried out on an ensemble of $\mathrm{GeVs}$, and we thus need to account for the random orientation of each emitter within the ND. We use weighted factors for the out-of-plane and in-plane components, $1 / 3$ and $2 / 3$, respectively, and determined the isotropic value $\alpha_{\text {iso }}=1 / 3 \alpha_{\perp}+2 / 3 \alpha_{\|}=0.35$. By combining the values for the ratio $\beta$ and $\alpha$, we obtain an estimate for the $\mathrm{QE}:^{.23,31}$

$\mathrm{QE}=k_{\mathrm{nr}} / k=(1-\beta) /\left(1-\alpha_{\mathrm{iso}}\right)=0.22 \pm 0.02$.

This value for $\mathrm{QE}$ is approximated, as the measurement could be optimized further. First, in the simulation, we do not take into account the refractive index of diamond, $n_{\text {diam }}=2.4$, which is higher than that of PMMA and glass $\left(n_{\text {glass }} \sim n_{\text {PMMA }} \sim 1.5\right)$.
A complete analysis would have to consider a total of three interfaces: diamond-air, PMMA-air, and diamond-PMMApotentially affecting the total radiative decay rate of the $\mathrm{GeV}$ centers. This problem could be alleviated by using, instead of glass and PMMA, a dielectric material (e.g., $\mathrm{TiO}_{2}$ ) with a refractive index closer to that of diamond $\left(n_{\mathrm{TiO} 2}=2.4\right)$, provided that the material does not mask the PL signal from the $\mathrm{GeV}$ emitters.

In addition, probing a single photon emitter rather than ensembles is desirable as it would remove the approximation associated with averaging over an ensemble of emitters. This however requires the photon extraction efficiency from a single $\mathrm{GeV}$ center to be high enough to be detected, practically, even after the deposition of a thick layer of dielectric material. Techniques such as bullseye cavities could in principle be employed. ${ }^{39}$ Alternatively, it should be possible to do shallow implantation of $\mathrm{GeVs}$ into bulk diamond, and then consequently overgrow a thick diamond layer to meet the requirement for two different dielectric environments. ${ }^{40}$

\section{Conclusions}

To conclude, we have characterized the properties of the $\mathrm{GeV}$ center in HPHT NDs, both in ensemble and single-emitter measurements. We found that both the position and linewidth of the GeV ZPL emission are stable due to symmetry protection of the $\mathrm{GeV}$ molecular configuration. Our results are compatible with previous reports of $\mathrm{SiV}$ and $\mathrm{GeV}$ centers in NDs. However, we find a relatively large spread of measured lifetimes $(\sim 6$ to $25 \mathrm{~ns})$. We attribute this variability to sample-dependent 
differences likely related to differences in the local photonic density of states. We performed BFP imaging of single $\mathrm{GeV}$ centers and combining experimental and theoretical data we reconstructed the orientation of each center, specifically the in-plane and out-of-plane angles of the emitting dipole(s) in the sample plane. Finally, we discussed an approximated method to measure the $\mathrm{QE}$ of an ensemble of $\mathrm{GeV}$ centers based on simple PL lifetime measurements. We determined the value of the $\mathrm{QE}$ of the $\mathrm{GeV}$ to be $(22 \pm 2) \%$. We noted that this value is an approximation and discussed ways to obtain a more precise estimate.

\section{Acknowledgments}

The authors thank Bundesministerium für Bildung und Forschung (BMBF) (NANO-FILM), Australian Research Council (ARC) (DP180100077, DP190101058, and DE180100810), and the Russian Science Foundation, Grant No. 19-12-00407 for financial support. We thank Dr. Alexander Solntsev and Dr. Sejeong Kim for their assistance during assembly of experimental setups and Dr. Benjamin Johnston at ANFF OptoFab for the micromachining of the sample slides.

\section{References}

1. M. Atatüre et al., "Material platforms for spin-based photonic quantum technologies," Nat. Rev. Mater. 3, 38-51 (2018).

2. D. D. Awschalom et al., "Quantum technologies with optically interfaced solid-state spins," Nat. Photonics 12(9), 516-527 (2018).

3. C. Bradac et al., "Quantum nanophotonics with group IV defects in diamond," Nat. Commun. 10, 5625 (2019).

4. M. Radulaski et al., "Nanodiamond integration with photonic devices," Laser Photonics Rev. 13(8), 1800316 (2019).

5. D. D. Sukachev et al., "Silicon-vacancy spin qubit in diamond: a quantum memory exceeding $10 \mathrm{~ms}$ with single-shot state readout," Phys. Rev. Lett. 119(22), 223602 (2017).

6. M. K. Bhaskar et al., "Quantum nonlinear optics with a germanium-vacancy color center in a nanoscale diamond waveguide," Phys. Rev. Lett. 118(22), 223603 (2017).

7. A. Sipahigil et al., "An integrated diamond nanophotonics platform for quantum-optical networks," Science 354, 847-850 (2016).

8. Y.-I. Sohn et al., "Controlling the coherence of a diamond spin qubit through its strain environment," Nat. Commun. 9, 2012 (2018).

9. B. Pingault et al., "Coherent control of the silicon-vacancy spin in diamond," Nat. Commun. 8, 15579 (2017).

10. T. T. Tran et al., "Anti-Stokes excitation of solid-state quantum emitters for nanoscale thermometry," Sci. Adv. 5(5), eaav9180 (2019).

11. A. E. Rugar et al., "Characterization of optical and spin properties of single tin-vacancy centers in diamond nanopillars," Phys. Rev. B 99(20), 205417 (2019).

12. L. J. Rogers et al., "Multiple intrinsically identical single-photon emitters in the solid state," Nat. Commun. 5, 4739 (2014).

13. L. J. Rogers et al., "Electronic structure of the negatively charged silicon-vacancy center in diamond," Phys. Rev. B 89(23), 235101 (2014).

14. N. Elke et al., "Single photon emission from silicon-vacancy colour centres in chemical vapour deposition nano-diamonds on iridium," New J. Phys. 13(2), 025012 (2011).

15. Y. Zhou et al., "Coherent control of a strongly driven silicon vacancy optical transition in diamond," Nat. Commun. 8, 14451 (2017).

16. T. Iwasaki et al., "Tin-vacancy quantum emitters in diamond," Phys. Rev. Lett. 119(25), 253601 (2017).

17. T. Iwasaki et al., "Germanium-vacancy single color centers in diamond," Sci. Rep. 5, 12882 (2015).
18. M. E. Trusheim et al., "Transform-limited photons from a tinvacancy spin in diamond," arXiv:1811.07777 (2019).

19. S. D. Tchernij et al., "Single-photon-emitting optical centers in diamond fabricated upon Sn implantation," ACS Photonics 4(10), 2580-2586 (2017).

20. G. Thiering and A. Gali, "Ab initio magneto-optical spectrum of group-IV vacancy color centers in diamond," Phys. Rev. X 8(2), 021063 (2018).

21. M. E. Trusheim et al., "Lead-related quantum emitters in diamond," Phys. Rev. B 99(7), 075430 (2019).

22. S. D. Tchernij et al., "Single-photon emitters in lead-implanted single-crystal diamond," ACS Photonics 5(12), 4864-4871 (2018).

23. V. G. Ralchenko et al., "Observation of the Ge-vacancy color center in microcrystalline diamond films," Bull. Lebedev Phys. Inst. 42(6), 165-168 (2015).

24. V. Sedov et al., "Growth of polycrystalline and single-crystal CVD diamonds with bright photoluminescence of $\mathrm{Ge}-\mathrm{V}$ color centers using germane $\mathrm{GeH}_{4}$ as the dopant source," Diamond Relat. Mater. 90, 47-53 (2018).

25. E. A. Ekimov et al., "Effect of Si, Ge and $\mathrm{Sn}$ dopant elements on structure and photoluminescence of nano- and microdiamonds synthesized from organic compounds," Diamond Relat. Mater. 93, 75-83 (2019).

26. Y. Zhou et al., "Direct writing of single germanium vacancy center arrays in diamond," New J. Phys. 20(12), 125004 (2018).

27. K. Bray et al., "Single crystal diamond membranes and photonic resonators containing germanium vacancy color centers," ACS Photonics 5(12), 4817-4822 (2018).

28. H. Siampour et al., "On-chip excitation of single germanium vacancies in nanodiamonds embedded in plasmonic waveguides," Light Sci. Appl. 7(1), 61 (2018).

29. W. Lukosz and R. E. Kunz, "Fluorescence lifetime of magnetic and electric dipoles near a dielectric interface," Opt. Commun. 20(2), 195-199 (1977).

30. X. Brokmann et al., "Measurement of the radiative and nonradiative decay rates of single CdSe nanocrystals through a controlled modification of their spontaneous emission," Phys. Rev. Lett. 93(10), 107403 (2004).

31. E. A. Ekimov et al., "High-pressure synthesis of nanodiamonds from adamantane myth or reality?" ChemNanoMat 4(3), 269273 (2018).

32. M. A. Lieb, J. M. Zavislan, and L. Novotny, "Single-molecule orientations determined by direct emission pattern imaging," J. Opt. Soc. Am. B 21(6), 1210-1215 (2004).

33. C. Hepp et al., "Electronic structure of the silicon vacancy color center in diamond," Phys. Rev. Lett. 112(3), 036405 (2014).

34. M. W. Doherty et al., "The nitrogen-vacancy colour centre in diamond," Phys. Rep. 528(1), 1-45 (2013).

35. Y.-K. Tzeng et al., "Vertical-substrate MPCVD epitaxial nanodiamond growth," Nano Lett. 17, 1489-1495 (2017).

36. E. Neu, M. Agio, and C. Becher, "Photophysics of single silicon vacancy centers in diamond: implications for single photon emission," Opt. Express 20(18), 19956-19971 (2012).

37. I. I. Vlasov et al., "Molecular-sized fluorescent nanodiamonds," Nat. Nanotechol. 9(1), 54-58 (2014).

38. C. Bradac et al., "Observation and control of blinking nitrogenvacancy centres in discrete nanodiamonds," Nat. Nanotechol. 5(5), 345-349 (2010).

39. L. Li et al., "Efficient photon collection from a nitrogen vacancy center in a circular bullseye grating," Nano Lett. 15(3), 1493-1497 (2015).

40. S. Castelletto et al., "Imaging and quantum-efficiency measurement of chromium emitters in diamond," Phys. Rev. Lett. 105(21), 217403 (2010).

Minh Nguyen is a PhD candidate at University of Technology Sydney (UTS). He obtained his BSc degree in nanotechnology, with honors, in 2017. 
Nguyen et al.: Photodynamics and quantum efficiency of germanium vacancy color centers...

Niko Nikolay is a PhD student at the Department of Physics of the Humboldt University of Berlin. He does research in quantum physics, solid state physics and optics.

Igor Aharonovich is a professor in the School of Mathematical and Physical Sciences at UTS. His group focuses on studies of quantum emitters in wide bandgap materials such as diamond and hexagonal boron nitride, nanofabrication of photonic devices, and studies of lightmatter interaction at the nanoscale.

Biographies of the other authors are not available. 